\title{
Multisystem Manifestations of Benign Ovarian Teratomas
}

\author{
William Murdoch, MD, Jill Sadoski, MD, and Frederick C. Rosin, MD
}

A 26-year-old woman presented with acute onset of right-sided pelvic pain and had a medical history significant for migraine headaches and polycystic ovarian disease. Ultrasonography demonstrated bilateral ovarian tumors, and the patient underwent laparoscopic removal of bilateral cystic teratomas. A literature review focused on similar presentations of teratomas revealed isolated cases of migraines and polycystic ovarian disease associated with teratomas and an increased risk for ovarian torsion. Our patient experienced complete resolution of her acute abdominal pain, as well as her long-standing headaches and hormonal symptoms, after removal of the teratomas. (J Am Board Fam Med 2014;27: 421-423.)

Keywords: Headache, Primary Health Care, Women's Health

Ms. $\mathrm{F}$ is a 26-year-old woman who is an established patient in our office. She presented with a severalhour history of intense, right-sided pelvic pain, which had started the previous evening immediately following intercourse. The pain was described as sharp and stabbing in nature and had subsided somewhat since the previous evening. Abdominal examination noted tenderness upon palpation in the right lower quadrant without rebound or guarding. Urine pregnancy testing was negative. The patient had previously visited an outside gynecologist for pelvic examinations. She was encouraged to see her gynecologist as soon as possible and was sent for an urgent ultrasound of the pelvis.

Ms. F had a medical history significant for polycystic ovarian syndrome (PCOS), migraine headaches, fibromyalgia, chronic fatigue, irritable bowel syndrome, and occipital neuralgias. Her diagnosis of PCOS was made 18 months before surgery on the basis of new-onset menstrual irregularities,

\footnotetext{
This article was externally peer reviewed.

Submitted 30 July 2013; revised 20 November 2013; accepted 25 November 2013.

From the Cittenton Family Medicine Residency, Wayne

State University, Rochester Hills, MI.

Funding: none.

Conflict of interest: none declared.

Corresponding author: William J. Murdoch, MD, Cittenton Family Medicine Residency, Wayne State University, 1135 W. University Dr., Ste 250, Rochester Hills, MI 48307 (E-mail: bmurdoch@med.wayne.edu).
}

acne, and weight gain. Hormonal studies nor ultrasonography of the pelvis were not performed at that time because her primary care physician and her gynecologist agreed on the clinical diagnosis. Ms. F was initiated on oral contraceptive pills and metformin at that point, which only modestly palliated her symptoms.

Notably, she had no history of pelvic inflammatory disease or endometriosis. She had a loop electrosurgical excision procedure 8 years earlier for advanced cervical dysplasia. Her medications at the time of the visit included ethinyl estradiol/drospirenone oral contraceptive, metformin, and vitamin D supplementation. She also used sumitriptan and an acetaminophen/sedative combination several times per week for headaches. She had no known drug allergies and no family history relevant to the presenting complaint. She was a former smoker and rarely drank alcohol.

Results of a complete blood count were unremarkable, as were various tumor markers (Table 1). Ultrasonography of the pelvis revealed bilateral complex cystic adnexal masses. The right-sided mass measured $4.2 \times 5.5 \mathrm{~cm}$ at its greatest diameter and arose from the right ovary. The left-sided mass measured $7 \times 7 \mathrm{~cm}$ at its greatest diameter, and the left ovary was not identifiable as a separate entity. There was no evidence of ovarian torsion.

When Ms. F presented to her gynecologist, her symptoms had evolved to bilateral pelvic soreness 
Table 1. Laboratory Data of a 26 Year-0ld Woman Presenting with Acute Right-Sided Pelvic Pain

\begin{tabular}{|c|c|c|}
\hline Laboratory Test & Normal Values & Ms. F's Results \\
\hline White blood cell count (thousands/ $\mu \mathrm{L}$ ) & $4.5-10.5$ & 7.0 \\
\hline Hemoglobin $(\mathrm{g} / \mathrm{dL})$ & $12.1-15.5$ & 15.5 \\
\hline Hematocrit (\%) & $35.0-45.0$ & 45.0 \\
\hline Platelet count (thousands $/ \mu \mathrm{L}$ ) & $130-400$ & 253 \\
\hline$\beta$-hCG quantitative (mIU/mL) & Clinically dependent & 0.9 \\
\hline Alpha Fetoprotein $(\mathrm{ng} / \mathrm{mL})$ & $0-15.0$ & 3.3 \\
\hline Cancer antigen 125 (units/mL) & $0-35.0$ & 16.0 \\
\hline Carbohydrate antigen 19-9 (units/mL) & $0-35$ & $<1$ \\
\hline Carcinoembryonic antigen $(\mathrm{ng} / \mathrm{mL})$ & $0-3.0$ & 2.4 \\
\hline Lactate dehydrogenase (units/L) & $50-200$ & 153 \\
\hline Inhibin A dimer $(\mathrm{pg} / \mathrm{mL})$ & Not reported & 23.6 \\
\hline Inhibin B (pg/mL) & Not reported & 20 \\
\hline
\end{tabular}

hCG, human chorionic gonadotropin; mIU, one million international units.

of a lesser intensity. She was scheduled for surgery and, 8 days after presentation to our office, underwent outpatient laparoscopic removal of bilateral adnexal masses. In the surgeon's postoperative opinion, the masses were consistent with ovarian dermoid tumors. Ms. F had an uncomplicated recovery from her surgery. Pathologic examination of the masses revealed benign cystic teratomas (otherwise known as dermoid tumors), including the classic findings of skin, hair, and tooth fragments contained within the tumors.

At the initial follow-up, Ms. F reported complete resolution of her pelvic pain. Interestingly, she also reported complete resolution of her longstanding symptoms of headaches and fatigue. She experienced her first documented migraine 3 years before her surgery, and their frequency was at least weekly during that period. At a 6-month follow-up, Ms. F still reported that she had not experienced a single headache since her surgery and that her fatigue was still much improved.

Following surgery, Ms. F chose to discontinue use of both medications. At the 6-month follow-up she also reported that her menstrual irregularities and acne had completely resolved despite not being treated for PCOS.

\section{Literature Review}

A literature review was performed using the following key words in a variety of combinations: ovarian, dermoid, teratoma, headache, polycystic ovarian syndrome (PCOS), torsion, acne, weight gain, and fibromyalgia. This resulted in over 250 results, which were reviewed and assessed for relevance. After the review, 5 articles remained and were used in this discussion.

The literature on the various clinical manifestations of ovarian teratomas is limited. Only one case study discussed headache associated with an ovarian teratoma; however, this case also discussed associated behavioral changes that were not seen in our patient. ${ }^{1}$ Because of the nonspecific nature of our patient's presenting symptoms, we included a case report describing PCOS with a cystic teratoma. ${ }^{2}$ This was done to further illustrate the idea that ovarian teratomas present with a broad spectrum of initial symptoms, which can lead to a delay in diagnosis or even misdiagnosis in some cases.

The theme throughout all the articles was the notion that clinical assessment is of limited value in the diagnosis of ovarian teratomas. Many of the presenting symptoms seen with ovarian teratomas overlap with other diseases, making imaging a critical part of the diagnostic workup. ${ }^{3}$ High clinical suspicion should warrant further testing and imaging studies. The initial diagnostic procedure of choice for ovarian teratomas is ultrasound examination. ${ }^{4}$ With that said, the nonspecific nature of the presenting symptoms associated with ovarian teratomas often lead to investigations with computed tomography and magnetic resonance imaging before ultrasound. ${ }^{3}$ To confirm the diagnosis of ovarian teratoma with imaging, intratumoral fat must be identified on the imaging modality. ${ }^{3}$

Ovarian teratomas can be described as mature or immature and consist of a variety of histologic types 
within the 3 germ layers, including ectoderm, mesoderm, and endoderm. ${ }^{3}$ They are associated with a variety of complications, with varying levels of severity. In this case, our patient experienced ovarian torsion as a result of the teratoma; therefore, the literature regarding this complication has been included. Ovarian torsion accounts for $16 \%$ of complications related to ovarian teratomas, and the most common risk factor for ovarian torsion is the presence of a dermoid cyst. ${ }^{4}$

\section{Discussion}

Ms. F's presentation seems unique based on our literature review. The case involving headache associated with a cystic teratoma included the presence of neural tissue in the teratoma. ${ }^{1}$ In our patient's case, no such tissue was seen during pathologic examination of her teratomas. That case also involved a much more rapid onset of neurological symptoms; our patient had a more indolent course of migraines for 3 years. Ovarian teratomas causing PCOS-like symptoms have been described previously, ${ }^{2}$ although it is worth mentioning that our patient experienced bilateral, not unilateral, teratomas. Whether this would affect the symptomatology of PCOS is unclear from the literature. The combination of PCOS and neurological symptoms caused by bilateral ovarian teratomas has not been previously described.

The natural history of cystic teratomas is quite variable, with at least $21 \%$ and as many as $64 \%$ discovered incidentally in asymptomatic patients. ${ }^{5}$ It is reasonable to surmise that our patient's teratomas were present for at least 3 years since her earliest attributable symptom was the onset of migraines 3 years before diagnosis.

Ovarian torsion caused by cystic teratoma is well described $^{4}$ and is the most common complication, with a reported incidence of $4.9 \% .^{5}$ Our patient's ovaries were not torsed on ultrasonographic or laparoscopic examination. At the time of those examinations, however, our patient's symptoms had progressed to a bilateral soreness, distinct from the intense, right-sided, stabbing pain she experienced in the initial hours of her presentation. It is interesting to wonder if her right ovary initially torsed but then "untorsed" before any tissue compromise.

Finally, a case series of 34 cystic teratomas included one case of coexistence with cervical squamous cell carcinoma in situ. ${ }^{5}$ Our patient had a loop electrosurgical excision procedure performed 8 years before presentation for advanced cervical dysplasia; it is possible that her teratomas were existent at that early date.

\section{Conclusion}

We present a case of a 26-year-old woman with a unique presentation of bilateral cystic teratomas manifesting as migraines and PCOS before overt presentation as pelvic pain. This case emphasizes the importance of a thorough workup when considering PCOS as a diagnosis, including ultrasonography of the pelvis. We also emphasize the variety of presentations of cystic teratomas, which often are asymptomatic but may present with hormonal or neurological symptoms and may cause ovarian torsion.

\section{References}

1. Sabin TD, Jednacz JA, Staats PN. Case records of the Massachusetts General Hospital. Case 26-2008. A 26-year-old woman with headache and behavioral changes. N Engl J Med 2008;359:842-53.

2. Cooke CW, McEvoy D, Wallach EE. Polycystic ovarian syndrome with unilateral cystic teratoma. Obstet Gynecol 1972;39:789-94.

3. Park SB, Kim JK, Kim KR, Cho KS. Imaging findings of complications and unusual manifestations of ovarian teratomas. Radiographics 2008;28:969-83.

4. Schwartz G. Radiological case of the month. Torsed ovary with a dermoid cyst. Arch Pediatr Adolesc Med 1998;152:503-4.

5. Ayhan A, Bukulmez O, Genc C, Karamursel BS, Ayhan A. Mature cystic teratomas of the ovary: case series from one institution over 34 years. Eur J Obstet Gynecol Reprod Biol 2000;88:153-7. 